\title{
Emergence of machine learning in the development of high entropy alloy and their prospects in advanced engineering applications
}

\author{
Nirmal Kumar Katiyar ${ }^{1,2} \cdot$ Gaurav Goel $^{1,2} \cdot$ Saurav Goel $^{1,2,3}$ (D)
}

Received: 7 April 2021 / Accepted: 8 June 2021 / Published online: 9 July 2021

(c) The Author(s) 2021

\begin{abstract}
The high entropy alloys have become the most intensely researched materials in recent times. They offer the flexibility to choose a large array of metallic elements in the periodic table, a combination of which produces distinctive desirable properties that are not possible to be obtained by the pristine metals. Over the past decade, a myriad of publications has inundated the aspects of materials synthesis concerning HEA. Hitherto, the practice of HEA development has largely relied on a trialand-error basis, and the hassles associate with this effort can be reduced by adopting a machine learning approach. This way, the "right first time" approach can be adopted to deterministically predict the right combination and composition of metallic elements to obtain the desired functional properties. This article reviews the latest advances in adopting machine learning approaches to predict and develop newer compositions of high entropy alloys. The review concludes by highlighting the newer applications areas that this accelerated development has enabled such that the HEA coatings can now potentially be used in several areas ranging from catalytic materials, electromagnetic shield protection and many other structural applications.
\end{abstract}

Keywords High entropy alloy (HEA) $\cdot$ Machine learning $\cdot$ Multicomponent alloy $\cdot$ Molecular dynamics $\cdot$ Density functional theory

\section{Introduction}

Chemical synthesis of high entropy alloys (HEAs) materials having different metallic compositions and resulting properties is a growing area of research. HEAs can readily be used across many sectors including defence, space, nuclear, energy generation and stealth applications. In 2004, two separate research groups based in the UK and Taiwan first coined the term "high entropy alloy" referring to HEA's ability to stabilise as a single solid solution phase based on the entropy of mixing $[1,2]$. The research momentum to discover newer HEA compositions has gained pace since then, and exceptional properties of HEAs are reported to date which includes magnetic properties [3, 4], catalytic

Saurav Goel

GoeLs@Lsbu.ac.uk

1 School of Engineering, London South Bank University, London SE10 AA, UK

2 School of Aerospace, Transport and Manufacturing, Cranfield University, Bedford MK43 0AL, UK

3 Shiv Nadar University, Gautam Budh Nagar 201314, India properties [5, 6], high conductivity, bio-compatibility and its ability to be additively manufactured [7] as well as high entropy ceramics [8], thermal barrier and bio-corrosion resistance [9], and more recently computational prediction works which have also started to emerge [10-12]. HEAs are reported to exhibit four core properties namely, high entropy, sluggish diffusion, lattice distortion and cocktail effects $[13,14]$. In a broader sense, the HEA family is like an apple tree where each apple represents a new field of study (see Fig. 1). The low-hanging fruit on this tree shown in Fig. 1a represents the application of HEA as structural materials with improved mechanical properties than the pristine materials. However, the top of this tree shows prospective biocompatible applications of HEA. However, this aspect will need further consideration, more intensive analysis and understanding and deeply study the long-term consequences of using multi-elements with each having different toxicity levels. Similarly, the field of high entropy polymer and superconductivity applications have started to gain pace, while the other areas shown in the apple tree are also being rigorously explored by various research groups around the globe. Cantor [15], one of the pioneers who proposed HEAs, used machine learning to predict that as many

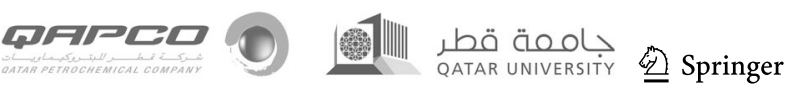


(a)


Fig. 1 a An apple tree represents the high entropy materials, and each apple represents the avenue of studies within high entropy materials; b development in HEAs materials steps towards artificial intelligence $(\mathrm{AI})$

as $\sim 10^{8}$ different types of HEA alloys can be developed from about 44 to 64 elements in the periodic table. The journey of HEAs from early days of development in 2004 based on largely empirical methods to the currently pursued machine learning approaches is highlighted in Fig. $1 b$.

Machine learning in materials design and discovery has been a fast-evolving field and flexibly adapts many databases to learn which includes surrogate models, iterative framework (adaptive design and learning), autonomous synthesis, atomistic simulations and text mining [16-18]. To date, machine learning has been successfully applied to predict the bulk metallic glasses [19], metal organic frameworks [20], thermoelectric materials [21], silicate glasses [22] and plant biomass [23] and design de novo materials and discover new mechanisms beyond intuition [24].

Science programs such as the Materials Genome Initiative leveraging the power of computation algorithms and physics theories based on classical Newton's and quantum laws are playing a significant role in the discovery of newer materials with predictive properties $[25,26]$. This has indeed become the fourth paradigm in the field of material science [27]. For successfully using machine learning to harness its full 
efficiency, it is important to set the basic rules and algorithms to be accurate [16]. Therefore, knowing the basics rules and sets of codification into equivalent computer language is a key step in applying machine learning whether it concerns the selection of materials, synthesis of materials, properties of materials, the interaction of an atom with other atoms or response of both.

The experimental results to date have provided a solid foundation to establish the chemistry of formulations of HEAs which helps guide the computational codes and algorithms. Simulations have long been used to instigate various phenomena such as the dynamics of biological systems [28], additive and subtractive manufacturing [29] and beyond. In the case of high entropy alloy synthesis, the concept of configurational entropy is well established [30, 31]. Based on these theoretical canons, commercial software's are developed to predict the phase, entropy and required temperatures of multicomponent alloys. One of the software THERMO-CALC has different versions of databases such as TCHEA4 and MOBHEA2 (https://www.thermocalc. com/solutions/by-material/high-entropy-alloys/), and it is continuously evolving. This software guides the first step in the predictive composition of HEAs together with the phase information. Subsequently, this prediction is realised by experimental synthesis using techniques such as arc melting, ball milling/cryomilling [32] and carbothermal shock [33]. However, coupled designing and application-based simulations of HEAs are somewhat in infancy, examples of which include phase prediction [34, 35], solid solution forming stage by ab initio approach [36], configurational entropy calculation [37] and microstructure prediction [38].

Amidst these continuously evolving developments, this article revisits the current literature and summarises the efforts made while using machine learning to develop newer compositions of high entropy alloys (HEAs) targeted to specific application areas.

\section{The thermodynamics behind the formation of HEAs}

According to thermodynamics, an alloy or a phase is stable when it possesses lower Gibbs free energy of mixing $\left(\Delta \mathrm{G}_{\text {mix }}\right)$ (see Eq. (1)). An increasing value of the term $T \Delta S_{\text {mix }}$ leads to a larger negative Gibbs free energy. According to the Boltzman hypothesis, shown in Eq. (2) [39], $\Delta \mathrm{S}_{\text {mix }}$ (entropy of mixing) depends on the arrangement of atoms in the crystalline lattice $(\omega)$ :

$$
\begin{aligned}
& \Delta G_{\text {mix }}=\Delta H_{\text {mix }}-T \Delta S_{\text {mix }} \\
& \Delta S_{\text {mix }}=k \ln (\omega)
\end{aligned}
$$

where $\mathrm{k}=$ Boltzmann constant and $\omega=\frac{\left(N_{A}+N_{B}\right) !}{N_{A} ! N_{B} !} ; \mathrm{N}_{\mathrm{A}}$ and $\mathrm{N}_{\mathrm{B}}$ are the atoms type species of mixing each other's. Thus, Eq. (2) can be rearranged by substitution, which gives

$\triangle S_{\text {mix }}=k \ln \left(\frac{\left(N_{A}+N_{B}\right) !}{N_{A} ! N_{B}}\right) g n$

Solving Eq. (3) by Sterling's approximation $\ln \mathrm{N} !=\mathrm{N}$ $\ln \mathrm{N}-\mathrm{N}$ yields

$\triangle S_{\operatorname{mix}}=k\left[\left(N_{A}+N_{B}\right) \ln \left(N_{A}+N_{B}\right)-N_{A}-N_{B}-N_{A} l n N_{A}-N_{B} \ln N_{B}+N_{A}+N_{B}\right]$

$\triangle S_{\text {mix }}=-k\left[N_{A}\left(\ln \frac{N_{A}}{N_{A}+N_{B}}\right)+N_{B}\left(\ln \frac{N_{B}}{N_{A}+N_{B}}\right)\right]$

$X_{A}=\frac{N_{A}}{N_{A}+N_{B}} ; X_{B}=\frac{N_{B}}{N_{A}+N_{B}} ; k \cdot N_{a}=R$

where $X_{A}$ and $X_{B}$ are the mole fraction of species $A$ and $\mathrm{B}, R$ is the universal gas constant and $N_{a}$ is the Avogadro's number.

$\Delta S_{m i x}=-R\left(X_{A} \ln X_{A}+X_{B} \ln X_{B}\right)$

Hence, Eq. (1) can be rearranged as

$\Delta G_{\text {mix }}=\Delta H_{\text {mix }}-T R\left(X_{A} \ln X_{A}+X_{B} \ln X_{B}\right)$

For the multicomponent (high entropy alloys) system, i.e. $N \geq 5$,

$\Delta S_{m i x}=-R\left(X_{A} \ln X_{A}+X_{B} \ln X_{B}+\cdots X_{E} \ln X_{E}\right)$

where $X_{A}, X_{B}, X_{C}, X_{D}$ and $X_{E}$ are mole fraction of species.

Hence, for equimolar systems $X_{A}=X_{B}=X_{C}=X_{D}=X_{E}$,

$\triangle S_{\text {mix }}=-R\left(X_{A} \ln X_{A}+\ldots \ldots \ldots X_{A} \ln X_{A}\right)$

Thus, for an equiatomic five elements system, each element fraction will be equal to 0.2

$\triangle S_{\text {mix }}=-R[\ln (0.2)]$

$\triangle S_{\text {mix }}=-R[\ln (5)]$

$\triangle S_{\text {mix }}=-R[\ln$ (Number of elements) $]$

Figure 2 shows the variation in the system's configuration entropy derived from Eq. (9) which can be categorised by high, medium and low entropy regions. A material composition having more than five elements in equiatomic proportion while forming a single phase is generally considered a high entropy alloy based on this notion.

\section{Phase prediction and electronic interactions}

The phase formation ability in alloys can be deduced through many ways such as valence electron configuration (VEC); electronegativity difference $(\Delta \chi)$ between the elements; enthalpy of mixing $\left(\Delta \mathrm{H}_{\text {mix }}\right)$; the entropy of mixing 


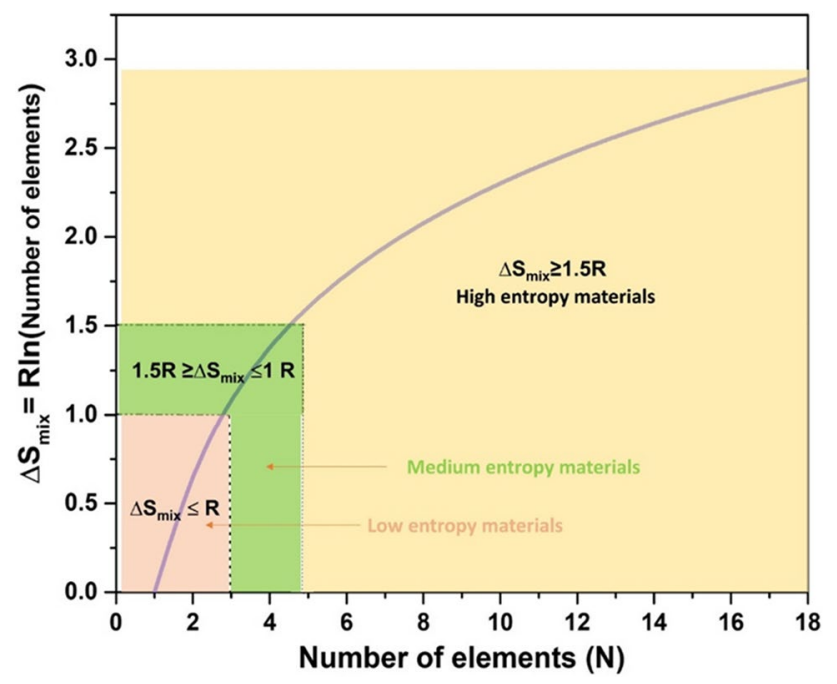

Fig. 2 Variation of entropy with the number of elements and their subdivision in low, medium and high entropy materials [40, 41]. Coloured regions in the chart represent the extent of anisotropy, so the pink colour is used to depict regions of low entropy, green for medium entropy and yellow for high entropy materials

$\left(\Delta \mathrm{S}_{\mathrm{mix}}\right)$; and alternative approaches such as CALPHAD, ab initio, density functional theory (DFT) and so on; albeit, the accuracy of prediction becomes important $[42,43]$. The high entropy alloy phase formation is not entirely dependent on any fixed or single approach or rules [44-48]. The phase formation is dependent on the selection parameters such as solid solution (SS) phase, intermetallic (IM) compound and mixed phase (SS + IM). Apart from these fundamental rules, machine learning can also be used to predict the alloy forming ability. Any machine learning model has a prediction certainty associated with it which is usually referred to as design space. Depending on the type of algorithm used, ML models may show prediction uncertainty which makes it important to benchmark an appropriate ML model at the beginning of the problem. As for HEA development, Huang et al. [43] used three machine learning models for comparison to identify the best phase prediction algorithms for HEAs using 401 experimental datasets. They used the K-nearest neighbour (KNN), support vector machine (SVM) and artificial neural network (ANN) methods. They observed that the ANN method showed $74.3 \%$ correctness in the phase prediction as opposed to the other two algorithms showing lower prediction accuracies. This aspect is shown in Fig. 3a-b highlighting five input parameters of ANN (VEC, $\Delta \mathrm{c}, \Delta \mathrm{H}_{\text {mix }}, \Delta \mathrm{S}_{\text {mix }}, \delta$ ) linked to the prediction accuracy. Figure $3 \mathrm{~b}(\mathrm{i}-\mathrm{iv})$ shows how individual input out of the five inputs is substituted one by one until the problem fully converges to aid to understand the influence of each input parameter in governing the output, which helps to establish the prediction error. Agarwal et al.
[49] employed an adaptive neuro-fuzzy interface system (ANFIS). It is a hybrid algorithm constructed by combining the artificial neural network (ANN) and fuzzy logic. They applied this scheme to seventeen elements, and this method revealed $84.21 \%$ prediction accuracy. Dai et al. [50] demonstrated the energy and force calculations utilising deep learning potential (DLP) and compare their results with DFT calculations for high entropy materials (HEM) such as $\left(\mathrm{Zr}_{0.2} \mathrm{Hf}_{0.2} \mathrm{Ti}_{0.2} \mathrm{Nb}_{0.2} \mathrm{Ta}_{0.2}\right) \mathrm{C}$. They suggested that the DLP fitting accurate in predicting the mechanical and thermal properties of monodispersed carbide and HEMs but were unsure about the accuracy of DLP in predicting the defect properties of the phases. Another research group used the machine learning strategy to indicate the number of stable phases in HEA by low dimensional descriptors [51].

As of now, only a few articles are available in this area, particularly going beyond phase prediction and evaluating the interaction phenomena between two atoms or nearest neighbour which seems to be holding promise in the future. Ching et al. [10] reported simulated data about the electronic structure and interatomic interactions between all elements in a biocompatible single alloy system. Their simulation concept is based on quantum mechanics, total bond order density (TBOD) and partial bond order density (PBOD). Figure $3 \mathrm{c}$ and $\mathrm{d}$ show a system of 250 atoms, atomic charges and contribution from each bond interaction in a TiNbTaZrMo alloy. It can be considered a breakthrough in high entropy alloy-based simulations for calculating their inner electronic structure and metallic bond interaction in metals' random solid solution. In yet another simulation-based research, the microstructure of $\mathrm{TaNbHfZr}$ alloy was obtained using combined Monte Carlo (MC) and molecular dynamics (MD) which revealed proximity or validation of experimental outcome (simulated microstructure vs transmission electron micrograph) shown in Fig. 3e [52]. It is therefore clear that this methodology holds high potentials for the discovery of new alloys and their interdisciplinary applications [34, 44, 53, 54].

\section{Machine learning to predict HEAs as structural materials}

Various structural materials used for different purposes such as bio-implants, aerospace components and bridge/ building construction possess unique properties such as corrosion resistance, biocompatibility, high specific strength and thermal stability. Machine learning utilises existing experimental data to predict newer properties of materials, as a function of different processing or composition inputs [18]. The discovery of Cantor alloy (FeCo$\mathrm{CrNiMn}$ ) and their deformation mechanism revealed the combination of fracture toughness and ductility due to 


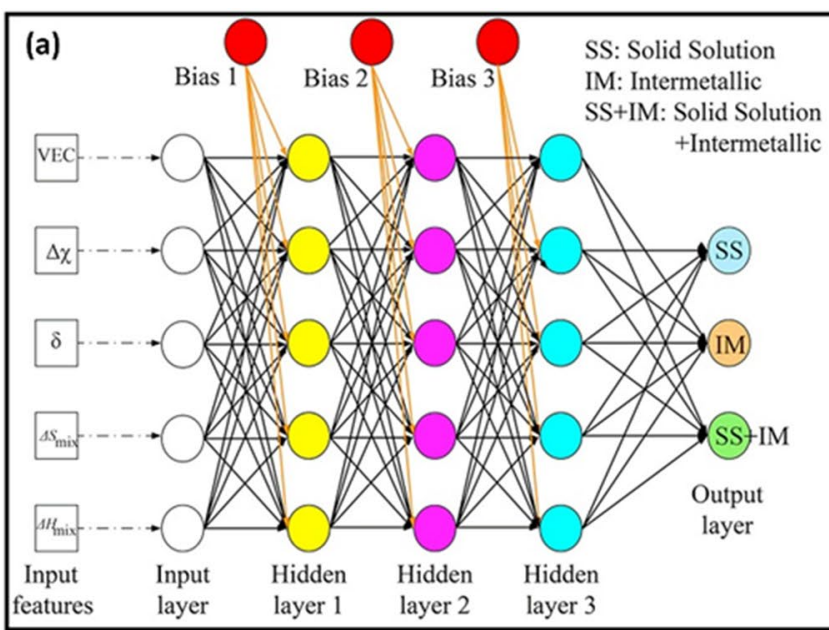

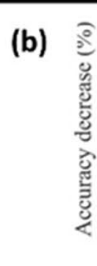
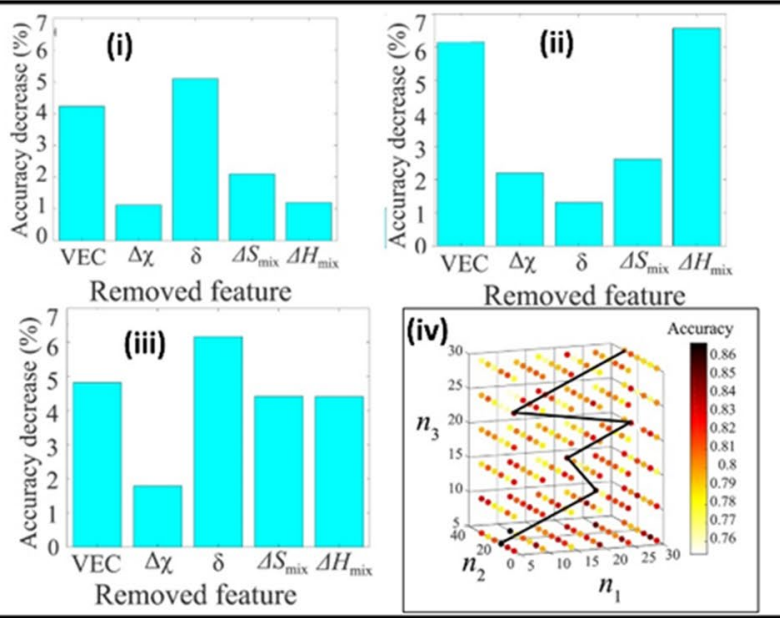
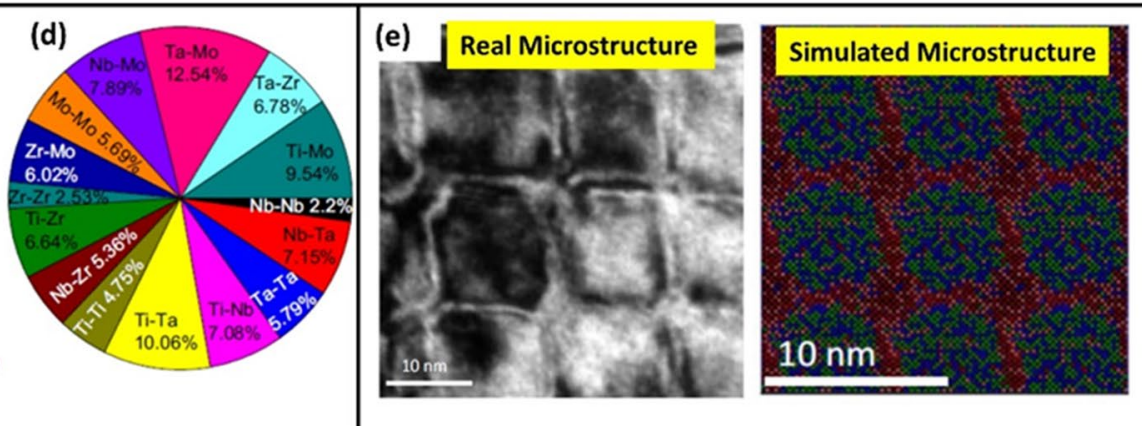

Fig. 3 a Artificial neural network with five input parameters [43]; b accuracy testing by removing one by one parameter out of the five parameters, (i) all three phases, (ii) SS + IM phase, (iii) SS + IM and IM phases with the multi-layer feed-forward neural network method and (iv) average testing accuracy of classifying two phases varies with the number of neurons in each hidden layer, denoted as n1, $\mathrm{n} 2$ and $\mathrm{n} 3$ for SS + IM [43]; c 250 atoms in 5 elements alloy partial charge on different atoms [10]; $\mathbf{d}$ percentage interaction between two bonds [10]; e HRTEM (high-resolution transmission electron microscopy) image of TaNbHfZr HEA; and simulated microstructure of TaNbHfZr HEA [52] (figures reprinted with permission from Elsevier Publishing Inc. and Nature Publishing Group) nano-twinning, which are induced during deformation at low temperature. For instance, the deformation behaviour and the balance of toughness and ductility found in natural materials like nacre [55] cannot be matched by the conventional alloys. Therefore, HEAs are so unique, and their development is gaining pace since their properties can be designed to obtain the desired properties. Furthermore, by tuning the entropy of mixing or Gibbs free energy, HEAs can be purpose designed to retain dual-phase (meta-stable phase) with desired toughness and ductility [56]. The traditional alloys and ceramics are always associated with a dichotomy of ductility and toughness [57]. A new concept, namely, transformation-induced plasticity-assisted, dualphase high entropy alloy (TRIPDP-HEA [56]), discusses the combined enhanced toughness and ductility behaviour of materials. It suggests that the two phases can be stabilised in a single high entropy alloy, where machine learning plays a major role. The non-equiatomic ratio of metallic elements in FeCoCrNiMn alloy alters their Gibbs free energy, allowing the FCC phase to be partially transformed (martensite transformation) into a hexagonal closed packed (HCP) phase. Therefore, the partial phase $\mathrm{HCP}$ in FCC improves the trans-grain and inter-grain resistance. The FCC and HCP phases can both have different deformation limits and show a large difference in their strengths for similar compositions. Therefore, we can say that the two bonded phases with one being softer compared to the other helps to improve materials strength, which makes the idea of applying machine learning quite exciting. A similar phenomenon is also observed in natural materials, for instance, in the nacre, the aragonite plates (95\%) bind with soft proteins (5\%) to enable them to balance the toughness and ductility [58]. Nacre's designs follow the hierarchical stacking of two different materials or phases, but in the dual-phase HEA materials, there is the absence of orderly distribution of two phases, as shown in Fig. 4a-d. The deformation behaviour of such materials is now being extensively explored. For example, Choi et al. [59] simulated the uniaxial tensile properties of singlecrystal nanowires of HEA ( $\mathrm{CoCrFeMnNi}$ ) containing

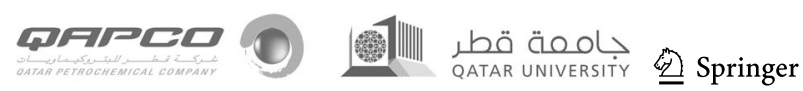


100,000 atoms ( $72 \AA \times 254 \AA \times 64 \AA$ ) and reported sluggish diffusion and micro twinning at cryogenic temperatures. Micro twinning plays a significant role in governing the excellent tensile properties observed at $77 \mathrm{~K}$, as shown in Fig. 4f-g. They believe that such HEAs have high dislocation mobility than conventional alloys due to the severe lattice distortion effect.

Recently, the non-equiatomic ratio of metallic elements capable to tune their transformation behaviour is newly emerging HEAs which are found to have shape memory effects at an extended range of temperatures [63] and hydrogen absorption with enhanced strength and ductility on loading [64]. It is believed that the extra stiffness in HEA stems from the lattice distortion brought by different sizes of atoms in the HEA. For example, a high entropy alloy $\mathrm{AlCoCrFeNi}$ has five different types of atoms with different electronegativity (Hume-Rothery rules). All these atom types are randomly distributed in a lattice structure which can vary depending on composition and parameters such as the interatomic distances and shear modulus anisotropy,
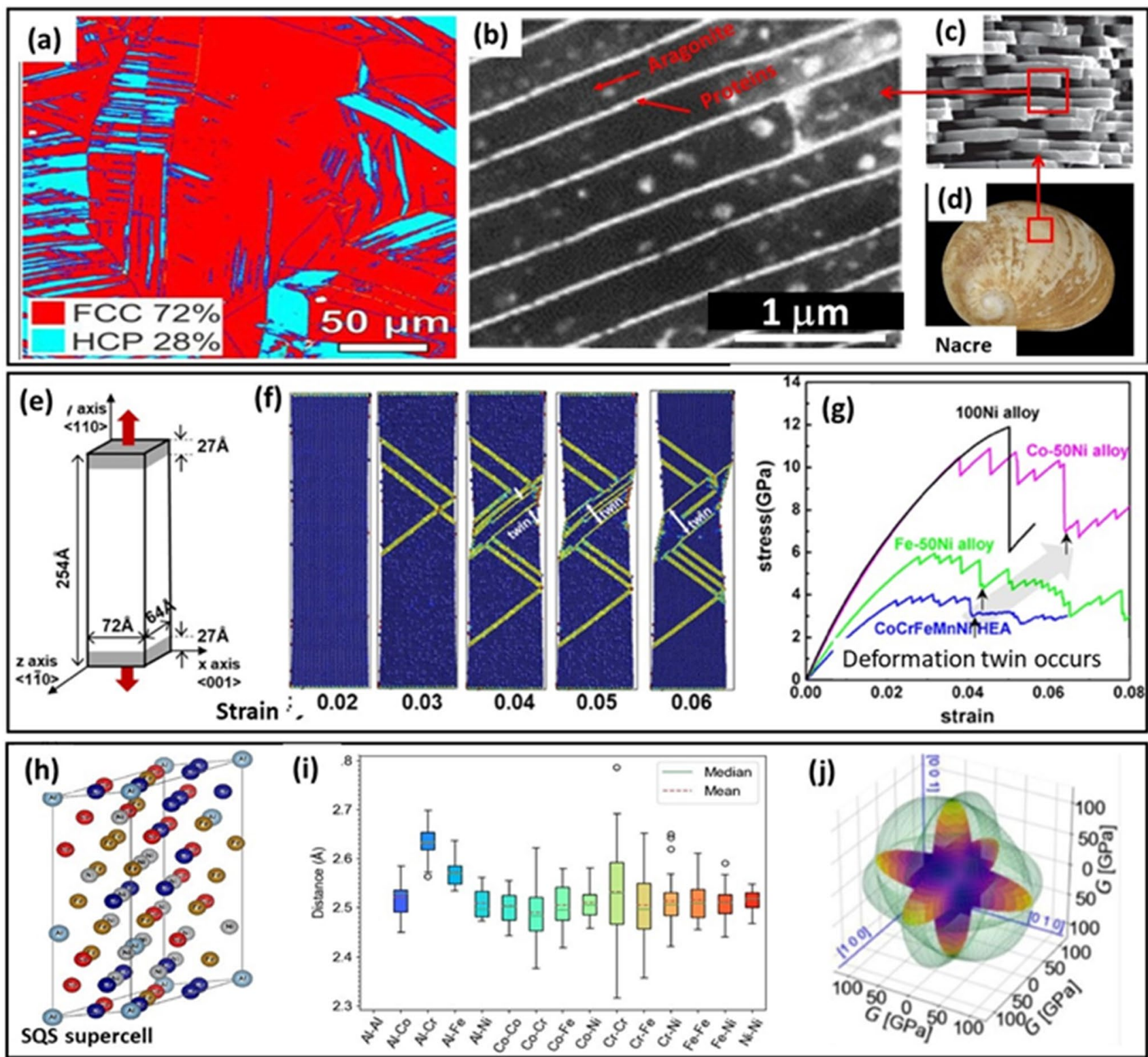

Fig. 4 a EDS mapping of $\mathrm{Co}_{20} \mathrm{Cr}_{20} \mathrm{Fe}_{34} \mathrm{Mn}_{20} \mathrm{Ni}_{6}$ (at\%) [60]; b TEM image of Nacre cross-section [61]; c SEM image of nacre cross section [61]; d Nacre image; e schematic uniaxial tensile test model; $\mathbf{f}$ simulated plastic deformation against strain of $\mathrm{CoCrFeMnNi}$ with 100,000 atoms model; g simulated stress-strain curve of $\mathrm{CoCr}$ -
FeMnNi HEA [59] at $0 \mathrm{~K}$; h SQS super cell of $\mathrm{Al}_{0.3} \mathrm{CoCrFeNi}$ HEA; $\mathbf{i}$ interatomic distances of different metallic elements in $\mathrm{Al}_{0.3} \mathrm{CoCrFeNi} ; \mathbf{j} 3 \mathrm{D}$ shear modulus anisotropy of $\mathrm{Al}_{0.3} \mathrm{CoCrFeNi}$ [62] (figures reprinted with permission from Elsevier Publishing Inc. and Nature Publishing Group)

\section{Springer}


computationally determined as shown in Fig. 4h-j. Kim et al. [62] studied elastic properties of $\mathrm{Al}_{0.3} \mathrm{CoCrFeNi} \mathrm{HEA}$ alloy by machine learning with validation experiments. Additionally, many research articles have started to examine defects dynamics in HEA alloys (FCC and BCC types) using MD simulations [65-70]. The defects in the alloys system play a major role in influencing the deformation mechanics and strength. The biggest challenge is to construct the small supercell with randomly distributed atoms to mimic each atom's real situation in a highly stoichiometric chemical environment. At present, various mathematical models are proposed to construct HEA supercell, but among these, the special quasirandom structural (SQS) model is the most popular. SQS uses a perfect random network around a given site to create two or more component periodic random alloy [71-73]. An alternative method, namely, the coherent potential approximation (CPA) [74], has started to emerge; however, this method does not consider the local lattice distortions, which is a major bottleneck. SQS model with DFT produces quite reliable results for short-range interatomic forces as it includes local lattice distortion [11] [72]. However, some uncertainty in results with a large supercell (different sizes of supercell) due to defects in the real samples [75] can be expected. Also, the force field or the potential function used in MD simulation faces challenges due to the variety of stoichiometry presented by the many atoms forming a single alloy. In this aspect, the use of machine learning for the development of more robust potential functions will continue to see a prosperous future.

\section{HEAs as functional materials}

This section provides a brief overview of the application of machine learning methods to discover HEAs for functional applications.

\subsection{Catalytic materials}

Nowadays, developing HEAs as catalyst materials has gained good popularity in chemical synthesis, particularly to reduce energy consumption and to synthesise new materials like reduced/oxidised organic products $\left(\mathrm{CO}_{2}\right.$ to $\mathrm{CH}_{4}$; $\mathrm{HCOOH}$ to $\mathrm{H}_{2}$, etc.) [6, 76-78]. HEAs comprise unique surfaces which can have multiple active energy level catalytic sites presented by multi-elements sitting over the surface (synergetic effect due to many random elements). Therefore, machine learning has high importance in designing synergistic catalytic sites by altering the composition for specific reactions (selectivity). The adsorbate adsorption over the catalyst surface can be determined using DFT simulations. Some theories determine the favourable catalyst sites for adsorbate by the $d$-band centre model and electron density of state (DOS) [6, 78, 79]. The high efficiency and stability of HEA properties evolved in the HEA alloys due to their $d$-band centre become closer to Fermi energy than pure metals, which plays a vital role in selectivity and efficiency [78]. Pedersen et al. [5] discovered AgAuCuPdPt as a new HEAs catalyst for the unbiased reduction of $\mathrm{CO}$ and $\mathrm{CO}_{2}$ using DFT. Their model predicted the favourable selectivity of the catalytic site of different metallic element combinations. Based on absorption energy on the top and the hollow site with different elements and their combination, a virtually designed catalyst suitable for the $\mathrm{CO}_{2}$ reduction reaction (CO2RR) was obtained with its response shown in Fig. 5a-c.

Yao et al. [80] have proposed a computationally entropydriven model to design multi-elements alloys nanoparticles (MEA-NPs) using conventionally immiscible elements (Ni, Ir, Mo, Fe, Cu, Ru, Cr, Pd, Co and Rh). However, the increasing entropy of mixing could make them miscible or single-phase alloy. The alloy elements designed were selectively based on $\mathrm{NH}_{3}$ decomposition $\left(\mathrm{H}_{2}\right.$ releases from $\mathrm{NH}_{3}$ as preferred catalytic site) from vast compositional space demonstrated in Fig. 5d. The combined Monte Carlo and MD simulation studies show that the alloy NPs endowed with thermal stability due to sluggish diffusion. The excellent catalytic activity of $\mathrm{NH}_{3}$ decomposition due to synergistic effects is shown in Fig. 5e. Among these, the RuRhCoNiIr alloy gave remarkable performance $\left(100 \% \mathrm{NH}_{3}\right.$ conversion), whereas a simple mix of the blend of these elements has not shown the same efficiency.

\subsection{Coatings}

Due to the high surface-to-volume ratio, coatings bring tremendous weight saving yet improved functionality and performance in various products. Ranging from biomedical implant to turbine blades, the use of coatings (both in thick and thin films) has long benefitted engineering components. The growth of the HEA films and their coalescence phenomenon can be very significant. In the very limited literature available on the spray coating of HEA, Xie et al. [81] have provided basic models of HEA deposition of $\mathrm{AlCrCoCuFeNi}$. They performed MD simulations to deposit this HEA on a silicon substrate to emulate magnetron sputtering. In these simulations, the substrate-coating ( $\mathrm{Si}-\mathrm{AlCrCoFeNi}$ interactions were established using Lennard-Jones potential. They claimed that their simulations showed good agreement with the experiments [82]. It may however be noted that the Cauchy pressure and interaction energies in a stoichiometric environment presented by the LJ potential could be erroneous which was the primary motivation behind the development of the embedded atom method (EAM) potential. So more accurate simulations will need more sophisticated potential functions or forcefields. Nevertheless, in their pioneering simulation study,

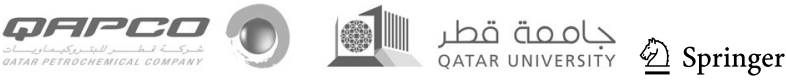



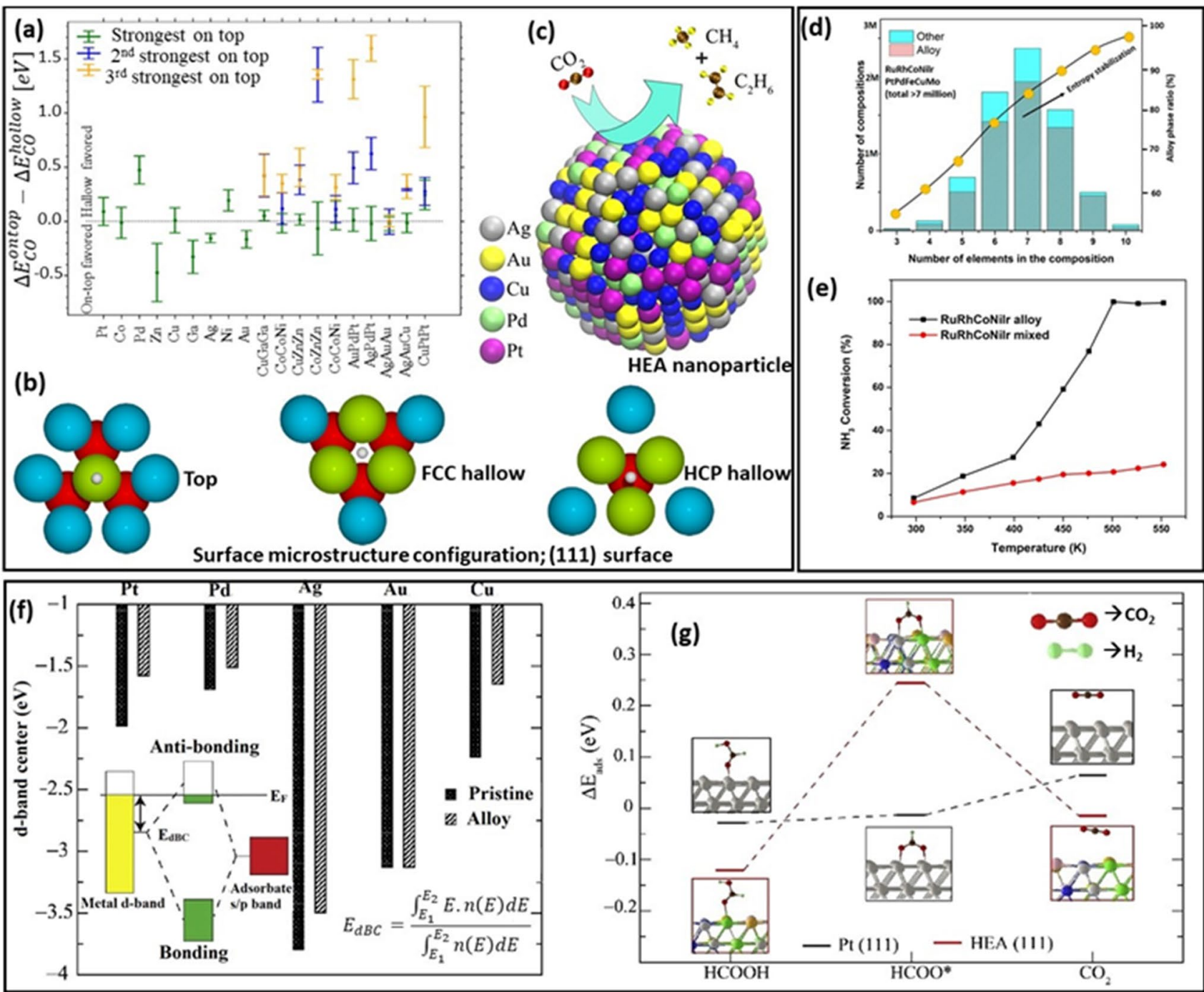

Fig. 5 a On top vs hollow site absorption energy of different compositions [6]; b representative of alloy surface structure configuration the (111) surface and their different sites [5]; c AgAuCuPdPt HEA nanoparticle [6]; $\mathbf{d}$ computational screening and entropy stabilisation;

they also highlighted pathline of the Fe atom to show that the movement of the atoms can be pronounced during the annealing process (see Fig. 6c). At lower temperatures, the movement of the $\mathrm{Fe}$ atom was highly localised, but it scaled exponentially with temperature. This type of simulation work is a very fertile area of research, and the use of robust potential functions is expected to shed light in this direction in the time to come.

\subsection{Electromagnetic wave shielding materials}

Electromagnetic (EM) wave pollution or interference is the core issue nowadays due to low-frequency waves omnipresent around us and the adverse effects they have on human health and sensitive electronic devices. Shielding from e $\mathrm{NH}_{3}$ conversion efficiency by RuRhCoNiIr HEA alloy and just similar blended elements [80] (figures reprinted with permissions from ACS publishing group, Elsevier Publishing Inc. and Science publishing Group)

electromagnetic interference works in two ways by absorption and reflection of EM. The incident EM waves get trapped by the complex microstructure over the material's surface (reflection loss) and absorbed when the available element's electric and magnetic dipole interacts with the incident electromagnetic wave. HEAs materials are promising in both ways as the metal surface can be easily altered due to metallic character and optimised to tune for the EM wave interaction. Usually, the material is developed to work across a broad range of EM absorption waves and low thickness materials are preferred. However, none of the currently available materials can work over a wide range (wavelength) to shield against the entire spectrum of the EM waves. High entropy alloy materials have promising potentials to work as electromagnetic wave shielding materials. There are some 

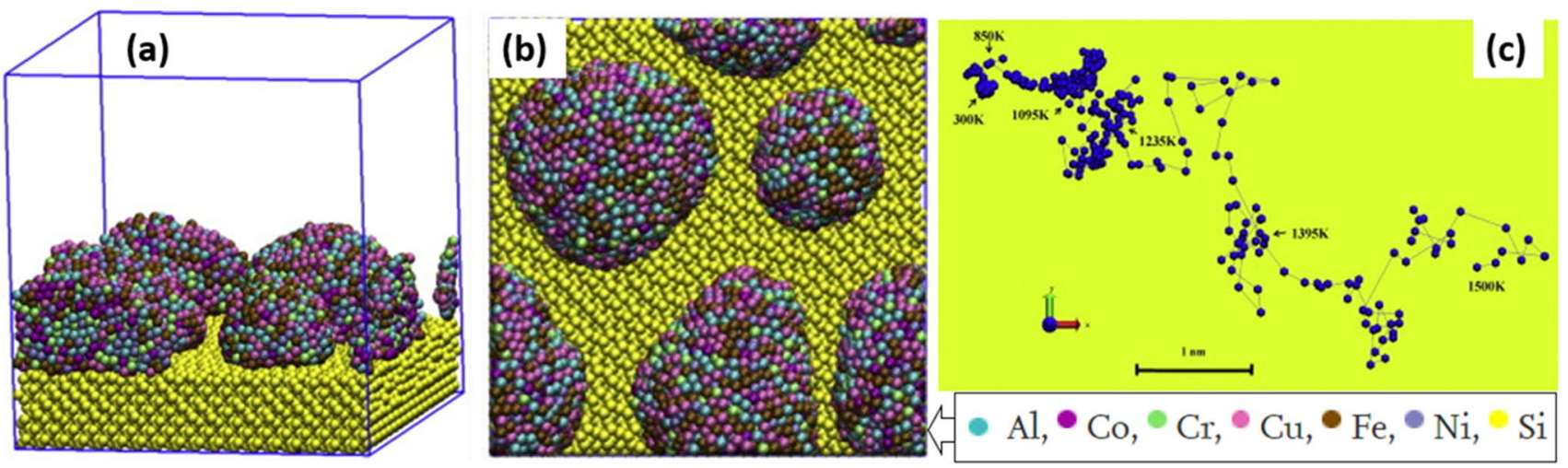

Fig. 6 Simulated film growth of HEA on Si substrate; a film side view, $\mathbf{b}$ film top view and $\mathbf{c}$ moving trajectory of Fe atom during annealing [81] (figures reprinted with permission from Elsevier Publishing Inc.)

experimental reports available on this front. One of the studies making use of MD reported by the nuclear energy university programme (NEUP) in the USA in 2014 [83] shows some details about it.

Interestingly, HEAs are also tested against radiation protection; for instance, the recrystallisation behaviour and vacancies and interstitial defects induced by a primary knock-on atom (PKA) were described to test the radiation tolerance of HEA [83]. In another study, the first principle calculation was used to study the irradiation resistance of $\mathrm{CoCrFeMnNi}$. It was found that the average binding energy of tri-vacancy in HEA is negative while tetra-vacancy binding energy is positive. Therefore, the instability of trivacancy or negative binding energy is responsible for the irradiation (ions or neutrons) resistance [84]. The irradiation induced defects in $\mathrm{NiCoCrFe} \mathrm{HEA} \mathrm{were} \mathrm{studied} \mathrm{through}$ MD simulations of modelled displacement cascades. In the PKA energies ranging from 10 to $50 \mathrm{keV}$, it was found that the higher defect recombination rate in the HEA, compared to that in pure $\mathrm{Ni}$ [85], contributes to enhanced damage tolerance.

\subsection{Miscellaneous properties and applications}

There are many functional applications of HEAs. This section provides a glimpse of probing various properties and applications of HEAs, which started with the prolific use of simulations. For example, the hydrogen absorption study of TiZrHfScMo high entropy alloy using DFT simulations revealed it as being an excellent hydrogen storage material. The hydrogenation is an exothermic reaction and $H$ bond with metal as a covalent bond, in which $T i$ and $S c$ lose an electron and Mo gain the electrons during absorption [86]. Another article shows Monte Carlo simulation of HEA to study the temperature-dependent chemical order of refractory metal (Mo-Nb-Ta-W) [87, 88]. Similarly, the reverse Monte Carlo structure modelling was also used to study the local lattice distortion in three HEAs, namely, TiVNb, TiVZrNb and TiVZrNbHf. It was reported that the increasing elemental size difference is the cause of local lattice distortion [89]. Ju et al. [90] investigated the melting behaviour of HEA (PtPdRhCo) nanoparticles using MEAM potential in MD. There are many more studies, which have used machine learning, such as the hydrogen absorption $\mathrm{Co}-\mathrm{Cr}-\mathrm{Fe}-\mathrm{Mn}-\mathrm{Ni}$ HEA alloy using the first principle calculation [91], inverse Hall-Petch relationship revealed in HEAs using atomistic simulations [92], superplasticity during martensite transformation in HEA nanowires [93] and diffusion of Cobalt in $\mathrm{Al}_{15} \mathrm{Sc}_{10} \mathrm{Hf}_{25} \mathrm{Ti}_{25} \mathrm{Zr}_{25}$ by ab initio calculations. The use of simulations in this area has yet to see many studies emerging in the time to come.

\section{Relevance of data reporting}

Machine learning and artificial intelligence rely on the source and accuracy of data. In this vein, numerous experimental research articles have appeared in peer-reviewed journals focussing on the preparation and characterisation of HEAs. However, many of these articles have used different conditions and processing parameters, which make their comparison a big challenge. Figure 7 shows the entire window of processing of HEA revealing that an incremental variation at any stage would lead to different microstructure and composition, which will make the comparison of HEA from two different labs challenging. Figure 7 also shows that most HEAs can have different compositions, i.e. different metal components or different stoichiometric ratios. In the second step of preparation, various processes can be used to prepare HEAs using distinct parameters, even having the same elements or stoichiometric ratio. Cryomilling process or mechanical milling has many input parameters such as duration of milling, milling parameters (rotation speed, infeed), ball-to-powder (BPR)

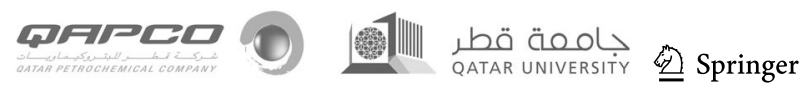


Fig. 7 A recipe for the preparation of HEA using different processing parameters

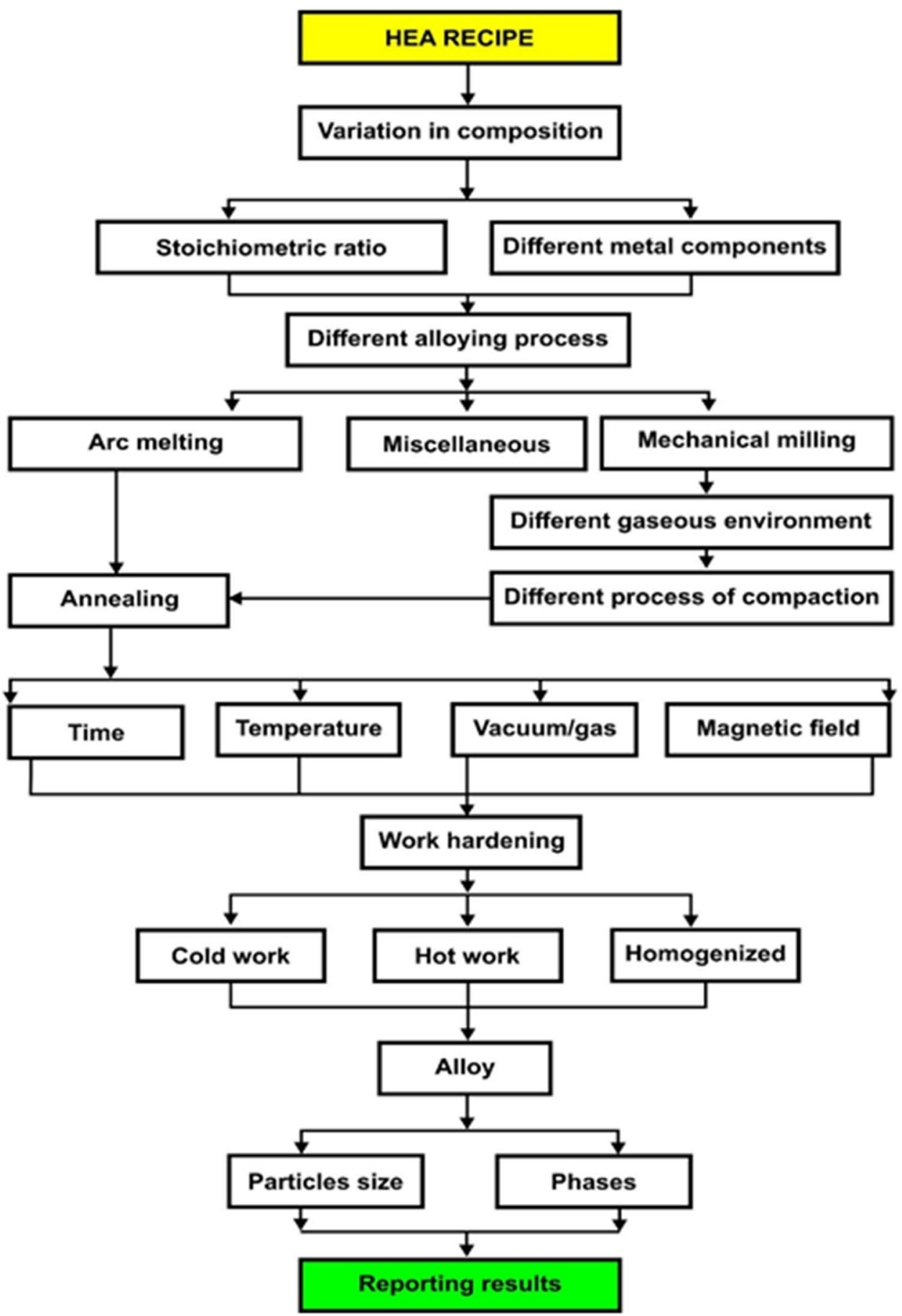

ratio etc. All these parameters can affect the properties of HEA due to different grain sizes. Also, a longer milling time can introduce debris from milling media. In the third step, the annealing or homogenisation also has various parameters. Also, the mechanical properties can vary with their cold/hard work. For example, in functional and catalytic applications, the components of HEAs, their stoichiometric ratio, particle size and extent of native/ pristine surface (oxides or other contaminants bind with the surface during preparation) can alter their catalytic performance. Therefore, the comparison of data from two different articles/lab is challenging, and it is an obstacle for machine learning. As such, there is an arduous need for an international standard for reporting the HEA with specified parameters. 


\section{Future challenges and prospects}

Machine learning for the development of HEAs is still in infancy, and to become a matured technology, many hurdles need to be crossed. Of these hurdles, the most important ones are the availability of open data, opensource software and a standard approach such as the one laid out in engineering ISO standards to make the comparison fair. The development of force field or potential energy functions for MD simulations is a complicated process due to many stoichiometric possibilities involving different atomic elements and the effect of thermodynamics parameters that need to be considered. Another challenge is to produce a supercell, although at present SQS method is being popularly used using software like Atomsk but it is not known whether it represents the realistic scenario that is observed during the experiments.

Open data repository of high entropy materials is yet to be developed in standardised format so that the open database can be used for different alloy simulations which will be useful to calibrate the predictions based on composition, processing conditions and their stabilisation in different phases.

Due to the involvement of multi-elements of chemical complexity, the density functional theory simulation requires huge computational power, limiting the model to very few atoms. However, scalable molecular dynamics on high-performance computers can now handle large systems, but a major bottleneck is the limited choices of the universal potential functions which can reliably depict the metallic bonding in the HEA. These limitations are obstacles to the development of applying AI and machine learning to study HEAs at present.

Funding All authors greatly acknowledge the financial support provided by the UKRI via Grants No. EP/L016567/1, EP/S013652/1, EP/S036180/1, EP/T001100/1 and EP/T024607/1; Royal Academy of Engineering via Grants No. IAPP18-19\295, TSP1332 and EXPP2021\1\277; EURAMET EMPIR A185 (2018); EU Cost Action (CA15102, CA18125, CA18224 and CA16235); and Newton Fellowship award from the Royal Society (NIF\R1\191571). We also acknowledge the financial support from the European Regional Development Funds (ERDF) sponsored A2i project at LSBU that have catalysed several industrial partnerships. Wherever applicable, the work made use of Isambard Bristol, UK supercomputing service accessed by Resource Allocation Panel (RAP) grant as well as ARCHER2 resources (Project e648).

Data availability As this a review paper, no additional data was generated.

Open Access This article is licensed under a Creative Commons Attribution 4.0 International License, which permits use, sharing, adaptation, distribution and reproduction in any medium or format, as long as you give appropriate credit to the original author(s) and the source, provide a link to the Creative Commons licence, and indicate if changes were made. The images or other third party material in this article are included in the article's Creative Commons licence, unless indicated otherwise in a credit line to the material. If material is not included in the article's Creative Commons licence and your intended use is not permitted by statutory regulation or exceeds the permitted use, you will need to obtain permission directly from the copyright holder. To view a copy of this licence, visit http://creativecommons.org/licenses/by/4.0/.

\section{References}

1. B. Cantor, I.T.H. Chang, P. Knight, A.J.B. Vincent, Microstructural development in equiatomic multicomponent alloys. Mater. Sci. Engg. A 375-377, 213-218 (2004). https://doi.org/ 10.1016/j.msea.2003.10.257

2. J.-W. Yeh, S.-K. Chen, S.-J. Lin, J.-Y. Gan, T.-S. Chin, T.-T. Shun, C.-H. Tsau, S.-Y. Chang, Nanostructured high-entropy alloys with multiple principal elements: novel alloy design concepts and outcomes. Adv. Engg. Mater. 6(5), 299-303 (2004). https://doi.org/10.1002/adem.200300567

3. Y. Zhang, T. Zuo, Y. Cheng, P.K. Liaw, High-entropy alloys with high saturation magnetization, electrical resistivity and malleability. Sci. Rep. 3(1), 1455 (2013). https://doi.org/10. 1038/srep01455

4. A. Marshal, K.G. Pradeep, D. Music, L. Wang, O. Petracic, J.M. Schneider, Combinatorial evaluation of phase formation and magnetic properties of $\mathrm{FeMnCoCrAl}$ high entropy alloy thin film library. Sci. Rep. 9(1), 7864 (2019). https://doi.org/ 10.1038/s41598-019-44351-8

5. J.K. Pedersen, T.A.A. Batchelor, A. Bagger, J. Rossmeisl, Highentropy alloys as catalysts for the $\mathrm{CO}_{2}$ and $\mathrm{CO}$ reduction reactions. ACS Catal. 10(3), 2169-2176 (2020). https://doi.org/10. 1021/acscatal.9b04343

6. S. Nellaiappan, N.K. Katiyar, R. Kumar, A. Parui, K.D. Malviya, K.G. Pradeep, A.K. Singh, S. Sharma, C.S. Tiwary, K. Biswas, High-entropy alloys as catalysts for the $\mathrm{CO}_{2}$ and $\mathrm{CO}$ reduction reactions: experimental realization. ACS Catal. 10(6), 3658-3663 (2020). https://doi.org/10.1021/acscatal.9b04302

7. C. Kenel, N.P.M. Casati, D.C. Dunand, 3D ink-extrusion additive manufacturing of $\mathrm{CoCrFeNi}$ high-entropy alloy micro-lattices. Nat. Commun. 10(1), 904 (2019). https://doi.org/10.1038/ s41467-019-08763-4

8. C. Oses, C. Toher, S. Curtarolo, High-entropy ceramics. Nat. Rev. Mater. 5(4), 295-309 (2020). https://doi.org/10.1038/ s41578-019-0170-8

9. G. Perumal, H.S. Grewal, M. Pole, L.V.K. Reddy, S. Mukherjee, H. Singh, G. Manivasagam, H.S. Arora, Enhanced biocorrosion resistance and cellular response of a dual-phase high entropy alloy through reduced elemental heterogeneity. ACS Appl. Bio Mater. 3(2), 1233-1244 (2020). https://doi.org/10.1021/acsabm.9b01127

10. W.-Y. Ching, S. San, J. Brechtl, R. Sakidja, M. Zhang, P.K. Liaw, Fundamental electronic structure and multiatomic bonding in 13 biocompatible high-entropy alloys. Npj Comput. Mater. 6(1), 45 (2020). https://doi.org/10.1038/s41524-020-0321-x

11. Z.H. Aitken, V. Sorkin, Y.-W. Zhang, Atomistic modeling of nanoscale plasticity in high-entropy alloys. J. Mater. Res. 34(9), 1509-1532 (2019). https://doi.org/10.1557/jmr.2019.50

12. L. Zhang, T. Hashimoto, J. Yan, Machinability exploration for high-entropy alloy $\mathrm{FeCrCoMnNi}$ by ultrasonic vibrationassisted diamond turning. CIRP Ann. (2021). https://doi.org/ 10.1016/j.cirp.2021.04.090

13. E.J. Pickering, N.G. Jones, High-entropy alloys: a critical assessment of their founding principles and future prospects. Int. Mater. Rev. 61(3), 183-202 (2016). https://doi.org/10.1080/ 09506608.2016.1180020

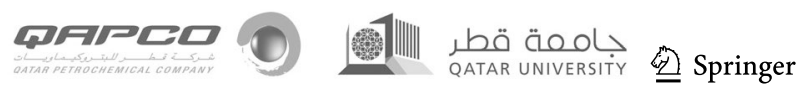


14. E.P. George, D. Raabe, R.O. Ritchie, High-entropy alloys. Nat. Rev. Mater. 4(8), 515-534 (2019). https://doi.org/10.1038/ s41578-019-0121-4

15. B. Cantor. Multicomponent high-entropy cantor alloys. Prog. Mater. Sci. 100754 (2020). https://doi.org/10.1016/j.pmatsci. 2020.100754 .

16. G. Pilania, Machine learning in materials science: from explainable predictions to autonomous design. Comput. Mater. Sci. 193, 110360 (2021). https://doi.org/10.1016/j.commatsci.2021.110360

17. Y. Pan, P. Zhou, Y. Yan, A. Agrawal, Y. Wang, D. Guo, S. Goel, New insights into the methods for predicting ground surface roughness in the age of digitalisation. Precis. Eng. 67, 393-418 (2021). https://doi.org/10.1016/j.precisioneng.2020.11.001

18. J. Schmidt, M.R.G. Marques, S. Botti, M.A.L. Marques, Recent advances and applications of machine learning in solid-state materials science. Npj Comput. Mater. 5(1), 83 (2019). https://doi.org/ 10.1038/s41524-019-0221-0

19. F. Ren, L. Ward, T. Williams, K.J. Laws, C. Wolverton, J. Hattrick-Simpers, A. Mehta, Accelerated discovery of metallic glasses through iteration of machine learning and high-throughput experiments. Sci. Adv. 4(4), eaaq1566 (2018). https://doi.org/10. 1126/sciadv.aaq1566

20. P. Halder, J.K. Singh, High-throughput screening of metal-organic frameworks for ethane-ethylene separation using the machine learning technique. Energy Fuels 34(11), 14591-14597 (2020). https://doi.org/10.1021/acs.energyfuels.0c03063

21. M. Einhorn, B.A.D. Williamson, D.O. Scanlon, Computational prediction of the thermoelectric performance of $\mathrm{LaZnOPn}(\mathrm{Pn}=$ P, As). J. Mater. Chem. A 8(16), 7914-7924 (2020). https://doi. org/10.1039/d0ta00690d

22. K. Yang, X. Xu, B. Yang, B. Cook, H. Ramos, N.M.A. Krishnan, M.M. Smedskjaer, C. Hoover, M. Bauchy, Predicting the Young's modulus of silicate glasses using high-throughput molecular dynamics simulations and machine learning. Sci. Rep. 9(1), 8739 (2019). https://doi.org/10.1038/s41598-019-45344-3

23. S. Zhou, K. Jin, M.J. Buehler. Understanding plant biomass via computational modeling. Adv. Mater. 2003206. https://doi.org/10. 1002/adma.202003206.

24. K. Guo, Z. Yang, C.-H. Yu, M.J. Buehler, Artificial intelligence and machine learning in design of mechanical materials. Mater. Horiz. (2021). https://doi.org/10.1039/D0MH01451F

25. K.T. Butler, D.W. Davies, H. Cartwright, O. Isayev, A. Walsh, Machine learning for molecular and materials science. Nature 559(7715), 547-555 (2018). https://doi.org/10.1038/ s41586-018-0337-2

26. M. Arita, D.R. Bowler, T. Miyazaki, Stable and efficient linear scaling first-principles molecular dynamics for $10000+$ atoms. J. Chem. Theory Comput. 10(12), 5419-5425 (2014). https://doi. org/10.1021/ct500847y

27. A. Agrawal, A. Choudhary, Perspective: Materials informatics and big data: realization of the "fourth paradigm" of science in materials science. APL Mater. 4(5), 053208 (2016). https://doi. org/10.1063/1.4946894

28. S. Wang, K. Fan, N. Luo, Y. Cao, F. Wu, C. Zhang, K.A. Heller, L. You, Massive computational acceleration by using neural networks to emulate mechanism-based biological models. Nat. Commun. 10(1), 4354 (2019). https://doi.org/10.1038/ s41467-019-12342-y

29. S. Goel, M. Knaggs, G. Goel, X.W. Zhou, H.M. Upadhyaya, V.K. Thakur, V. Kumar, G. Bizarri, A. Tiwari, A. Murphy, A. Stukowski, A. Matthews, Horizons of modern molecular dynamics simulation in digitalized solid freeform fabrication with advanced materials. Mater. Today Chem. 18, 100356 (2020). https://doi.org/ 10.1016/j.mtchem.2020.100356
30. C. Zhang, F. Zhang, S. Chen, W. Cao, Computational thermodynamics aided high-entropy alloy design. JOM 64(7), 839-845 (2012). https://doi.org/10.1007/s11837-012-0365-6

31. A. Ferrari, B. Dutta, K. Gubaev, Y. Ikeda, P. Srinivasan, B. Grabowski, F. Körmann, Frontiers in atomistic simulations of high entropy alloys. J. Appl. Phys. 128(15), 150901 (2020). https://doi. org/10.1063/5.0025310

32. N. Kumar, C.S. Tiwary, K. Biswas, Preparation of nanocrystalline high-entropy alloys via cryomilling of cast ingots. J. Mater. Sci. 53(19), 13411-13423 (2018). https://doi.org/10.1007/ s10853-018-2485-Z

33. Y. Yao, Z. Huang, P. Xie, S.D. Lacey, R.J. Jacob, H. Xie, F. Chen, A. Nie, T. Pu, M. Rehwoldt, D. Yu, M.R. Zachariah, C. Wang, R. Shahbazian-Yassar, J. Li, L. Hu, Carbothermal shock synthesis of high-entropy-alloy nanoparticles. Science 359(6383), 1489-1494 (2018). https://doi.org/10.1126/science. aan5412

34. Z. Zhou, Y. Zhou, Q. He, Z. Ding, F. Li, Y. Yang, Machine learning guided appraisal and exploration of phase design for high entropy alloys. Npj Comput. Mater. 5(1), 128 (2019). https://doi. org/10.1038/s41524-019-0265-1

35. L.J. Santodonato, P.K. Liaw, R.R. Unocic, H. Bei, J.R. Morris, Predictive multiphase evolution in Al-containing high-entropy alloys. Nat. Commun. 9(1), 4520 (2018). https://doi.org/10.1038/ s41467-018-06757-2

36. Y. Lederer, C. Toher, K.S. Vecchio, S. Curtarolo, The search for high entropy alloys: a high-throughput ab-initio approach. Acta Mater. 159, 364-383 (2018). https://doi.org/10.1016/j.actamat. 2018.07.042

37. J. Zhang, X. Liu, S. Bi, J. Yin, G. Zhang, M. Eisenbach, Robust data-driven approach for predicting the configurational energy of high entropy alloys. Mater. Des. 185, 108247 (2020). https://doi. org/10.1016/j.matdes.2019.108247

38. J. Li, Q. Fang, P.K. Liaw, Microstructures and properties of highentropy materials: modeling, simulation, and experiments. Adv. Engg. Mater. 23(1), 2001044 (2021). https://doi.org/10.1002/ adem. 202001044

39. G.E. Dieter, Mechanical Metallurgy (McGraw-Hill, New York, 1986)

40. K.. Biswas, N. Kumar, The effect of configurational entropy of mixing on the design and development of novel materials. Proc. Indian Natl. Sci. Acad. 86(3), 8 (2020). https://doi.org/10.16943/ ptinsa/2019/49674

41. J.-W. Yeh, Overview of High-Entropy Alloys, in High-entropy alloys: fundamentals and applications. ed. by M.C. Gao, J.-W. Yeh, P.K. Liaw, Y. Zhang (Springer International Publishing, Cham, 2016), pp. 1-19

42. N. Islam, W. Huang, H.L. Zhuang, Machine learning for phase selection in multi-principal element alloys. Comput. Mater. Sci. 150, 230-235 (2018). https://doi.org/10.1016/j.commatsci.2018. 04.003

43. W. Huang, P. Martin, H.L. Zhuang, Machine-learning phase prediction of high-entropy alloys. Acta Mater. 169, 225-236 (2019). https://doi.org/10.1016/j.actamat.2019.03.012

44. Y. Ikeda, B. Grabowski, F. Körmann, Ab initio phase stabilities and mechanical properties of multicomponent alloys: a comprehensive review for high entropy alloys and compositionally complex alloys. Mater. Charact. 147, 464-511 (2019). https://doi.org/ 10.1016/j.matchar.2018.06.019

45. S.Y. Lee, S. Byeon, H.S. Kim, H. Jin, S. Lee, Deep learning-based phase prediction of high-entropy alloys: optimization, generation, and explanation. Mater. Des. 197, 109260 (2021). https://doi.org/ 10.1016/j.matdes.2020.109260

46. C. Wen, Y. Zhang, C. Wang, D. Xue, Y. Bai, S. Antonov, L. Dai, T. Lookman, Y. Su, Machine learning assisted design of high entropy 
alloys with desired property. Acta Mater. 170, 109-117 (2019). https://doi.org/10.1016/j.actamat.2019.03.010

47. L. Zhang, H. Chen, X. Tao, H. Cai, J. Liu, Y. Ouyang, Q. Peng, Y. Du, Machine learning reveals the importance of the formation enthalpy and atom-size difference in forming phases of high entropy alloys. Mater. Des. 193, 108835 (2020). https://doi.org/ 10.1016/j.matdes.2020.108835

48. Y. Zhang, C. Wen, C. Wang, S. Antonov, D. Xue, Y. Bai, Y. Su, Phase prediction in high entropy alloys with a rational selection of materials descriptors and machine learning models. Acta Mater. 185, 528-539 (2020). https://doi.org/10.1016/j.actamat.2019.11. 067

49. A. Agarwal, A.K. Prasada Rao, Artificial intelligence predicts body-centered-cubic and face-centered-cubic phases in highentropy alloys. JOM 71(10), 3424-3432 (2019). https://doi.org/ 10.1007/s 11837-019-03712-4

50 F.-Z. Dai, B. Wen, Y. Sun, H. Xiang, Y. Zhou, Theoretical prediction on thermal and mechanical properties of high entropy ( $\mathrm{Zr} 0.2 \mathrm{Hf} 0.2 \mathrm{Ti0}$.2Nb0.2Ta0.2)C by deep learning potential. J. Mater. Sci. Technol. 43, 168-174 (2020). https://doi.org/10. 1016/j.jmst.2020.01.005

51. D. Dai, T. Xu, X. Wei, G. Ding, Y. Xu, J. Zhang, H. Zhang, Using machine learning and feature engineering to characterize limited material datasets of high-entropy alloys. Comput. Mater. Sci. 175, 109618 (2020). https://doi.org/10.1016/j.commatsci. 2020.109618

52. S. Mishra, S. Maiti, B.S. Dwadasi, B. Rai, Realistic microstructure evolution of complex Ta-Nb-Hf-Zr high-entropy alloys by simulation techniques. Sci. Rep. 9(1), 16337 (2019). https://doi.org/10. 1038/s41598-019-52170-0

53. T. Kostiuchenko, F. Körmann, J. Neugebauer, A. Shapeev, Impact of lattice relaxations on phase transitions in a high-entropy alloy studied by machine-learning potentials. Npj Comput. Mater. 5(1), 55 (2019). https://doi.org/10.1038/s41524-019-0195-y

54. M.C. Gao, P. Gao, J.A. Hawk, L. Ouyang, D.E. Alman, M. Widom, Computational modeling of high-entropy alloys: Structures, thermodynamics and elasticity. J. Mater. Res. 32(19), 36273641 (2017). https://doi.org/10.1557/jmr.2017.366

55. H.-L. Gao, S.-M. Chen, L.-B. Mao, Z.-Q. Song, H.-B. Yao, H. Cölfen, X.-S. Luo, F. Zhang, Z. Pan, Y.-F. Meng, Y. Ni, S.-H. Yu, Mass production of bulk artificial nacre with excellent mechanical properties. Nat. Commun. 8(1), 287 (2017). https://doi.org/10. 1038/s41467-017-00392-Z

56. Z. Li, K.G. Pradeep, Y. Deng, D. Raabe, C.C. Tasan, Metastable high-entropy dual-phase alloys overcome the strength-ductility trade-off. Nature 534(7606), 227-230 (2016). https://doi.org/10. 1038/nature 17981

57. M.E. Launey, R.O. Ritchie, On the fracture toughness of advanced materials. Adv. Mater. 21(20), 2103-2110 (2009). https://doi.org/ 10.1002/adma.200803322

58. G. Du, A. Mao, J. Yu, J. Hou, N. Zhao, J. Han, Q. Zhao, W. Gao, T. Xie, H. Bai, Nacre-mimetic composite with intrinsic selfhealing and shape-programming capability. Nat. Commun. 10(1), 800 (2019). https://doi.org/10.1038/s41467-019-08643-x

59. W.-M. Choi, Y.H. Jo, S.S. Sohn, S. Lee, B.-J. Lee, Understanding the physical metallurgy of the $\mathrm{CoCrFeMnNi}$ high-entropy alloy: an atomistic simulation study. Npj Comput. Mater. 4(1), 1 (2018). https://doi.org/10.1038/s41524-017-0060-9

60. Z. Li, F. Körmann, B. Grabowski, J. Neugebauer, D. Raabe, $\mathrm{Ab}$ initio assisted design of quinary dual-phase high-entropy alloys with transformation-induced plasticity. Acta Mater. 136, 262-270 (2017). https://doi.org/10.1016/j.actamat.2017.07.023

61. F. Song, A.K. Soh, Y.L. Bai, Structural and mechanical properties of the organic matrix layers of nacre. Biomaterials 24(20), 36233631 (2003). https://doi.org/10.1016/S0142-9612(03)00215-1
62. G. Kim, H. Diao, C. Lee, A.T. Samaei, T. Phan, M. de Jong, K. An, D. Ma, P.K. Liaw, W. Chen, First-principles and machine learning predictions of elasticity in severely lattice-distorted highentropy alloys with experimental validation. Acta Mater. 181, 124-138 (2019). https://doi.org/10.1016/j.actamat.2019.09.026

63. J.I. Lee, K. Tsuchiya, W. Tasaki, H.S. Oh, T. Sawaguchi, H. Murakami, T. Hiroto, Y. Matsushita, E.S. Park, A strategy of designing high-entropy alloys with high-temperature shape memory effect. Sci. Rep. 9(1), 13140 (2019). https://doi.org/10.1038/ s41598-019-49529-8

64. H. Luo, Z. Li, D. Raabe, Hydrogen enhances strength and ductility of an equiatomic high-entropy alloy. Sci. Rep. 7(1), 9892 (2017). https://doi.org/10.1038/s41598-017-10774-4

65. S.I. Rao, C. Woodward, T.A. Parthasarathy, O. Senkov, Atomistic simulations of dislocation behavior in a model FCC multicomponent concentrated solid solution alloy. Acta Mater. 134, 188-194 (2017). https://doi.org/10.1016/j.actamat.2017.05.071

66. S.I. Rao, C. Varvenne, C. Woodward, T.A. Parthasarathy, D. Miracle, O.N. Senkov, W.A. Curtin, Atomistic simulations of dislocations in a model BCC multicomponent concentrated solid solution alloy. Acta Mater. 125, 311-320 (2017). https://doi.org/10.1016/j. actamat.2016.12.011

67 A. Sharma, G. Balasubramanian, Dislocation dynamics in $\mathrm{A} 10.1 \mathrm{CoCrFeNi}$ high-entropy alloy under tensile loading. Intermetallics 91, 31-34 (2017). https://doi.org/10.1016/j.intermet. 2017.08.004

68. C. Varvenne, G.P.M. Leyson, M. Ghazisaeidi, W.A. Curtin, Solute strengthening in random alloys. Acta Mater. 124, 660-683 (2017). https://doi.org/10.1016/j.actamat.2016.09.046

69. C. Varvenne, A. Luque, W.A. Curtin, Theory of strengthening in fcc high entropy alloys. Acta Mater. 118, 164-176 (2016). https:// doi.org/10.1016/j.actamat.2016.07.040

70. Y. Afkham, M. Bahramyan, R.T. Mousavian, D. Brabazon, Tensile properties of $\mathrm{AlCrCoFeCuNi}$ glassy alloys: a molecular dynamics simulation study. Mater. Sci. Eng. A 698, 143-151 (2017). https:// doi.org/10.1016/j.msea.2017.05.057

71. A. Zunger, S.H. Wei, L.G. Ferreira, J.E. Bernard, Special quasirandom structures. Phys. Rev. Lett. 65(3), 353-356 (1990). https:// doi.org/10.1103/PhysRevLett.65.353

72. H. Song, F. Tian, Q.-M. Hu, L. Vitos, Y. Wang, J. Shen, N. Chen, Local lattice distortion in high-entropy alloys. Phys. Rev. Mater. 1(2), 023404 (2017). https://doi.org/10.1103/PhysRevMaterials. 1.023404

73. K. Okhotnikov, T. Charpentier, S. Cadars, Supercell program: a combinatorial structure-generation approach for the local-level modeling of atomic substitutions and partial occupancies in crystals. J. Cheminform. 8(1), 17 (2016). https://doi.org/10.1186/ s13321-016-0129-3

74. L. Vitos, I.A. Abrikosov, B. Johansson, Anisotropic lattice distortions in random alloys from first-principles theory. Phys. Rev. Lett. 87(15), 156401 (2001). https://doi.org/10.1103/PhysRevLett.87. 156401

75. L.-Y. Tian, Q.-M. Hu, R. Yang, J. Zhao, B. Johansson, L. Vitos, Elastic constants of random solid solutions by SQS and CPA approaches: the case of fcc Ti-Al. J. Phys.: Condens. Matter 27(31), 315702 (2015). https://doi.org/10.1088/0953-8984/27/ $31 / 315702$

76. A. Amiri, R. Shahbazian-Yassar, Recent progress of high-entropy materials for energy storage and conversion. J. Mater. Chem. A (2020). https://doi.org/10.1039/D0TA09578H

77. T.A.A. Batchelor, J.K. Pedersen, S.H. Winther, I.E. Castelli, K.W. Jacobsen, J. Rossmeisl, High-entropy alloys as a discovery platform for electrocatalysis. Joule 3(3), 834-845 (2019). https://doi. org/10.1016/j.joule.2018.12.015

78. N.K. Katiyar, S. Nellaiappan, R. Kumar, K.D. Malviya, K.G. Pradeep, A.K. Singh, S. Sharma, C.S. Tiwary, K. Biswas, Formic 
acid and methanol electro-oxidation and counter hydrogen production using nano high entropy catalyst. Mater. Today Eng. 16, 100393 (2020). https://doi.org/10.1016/j.mtener.2020.100393

79. M.T. Gorzkowski, A. Lewera, Probing the limits of d-band center theory: electronic and electrocatalytic properties of Pd-shellPt-core nanoparticles. J. Phy. Chem. C 119(32), 18389-18395 (2015). https://doi.org/10.1021/acs.jpcc.5b05302

80. Y. Yao, Z. Liu, P. Xie, Z. Huang, T. Li, D. Morris, Z. Finfrock, J. Zhou, M. Jiao, J. Gao, Y. Mao, J. Miao, P. Zhang, R. Shahbazian-Yassar, C. Wang, G. Wang, L. Hu, Computationally aided, entropy-driven synthesis of highly efficient and durable multielemental alloy catalysts. Sci. Adv 6(11), eaaz0510 (2020). https:// doi.org/10.1126/sciadv.aaz0510

81. L. Xie, P. Brault, A.-L. Thomann, J.-M. Bauchire, AlCoCrCuFeNi high entropy alloy cluster growth and annealing on silicon: a classical molecular dynamics simulation study. Appl. Surf. Sci. 285, 810-816 (2013). https://doi.org/10.1016/j.apsusc.2013.08.133

82. L. Xie, P. Brault, A.-L. Thomann, X. Yang, Y. Zhang, G. Shang, Molecular dynamics simulation of $\mathrm{Al}-\mathrm{Co}-\mathrm{Cr}-\mathrm{Cu}-\mathrm{Fe}-\mathrm{Ni}$ high entropy alloy thin film growth. Intermetallics 68, 78-86 (2016). https://doi.org/10.1016/j.intermet.2015.09.008

83. K. Peter, Liaw, Radiation Behavior of High-Entropy Alloys for Advanced Reactors (The University of Tennessee, Tennessee, 2014)

84. Q. Xu, H.Q. Guan, Z.H. Zhong, S.S. Huang, J.J. Zhao, Irradiation resistance mechanism of the $\mathrm{CoCrFeMnNi}$ equiatomic highentropy alloy. Sci. Rep. 11(1), 608 (2021). https://doi.org/10.1038/ s41598-020-79775-0

85. Y. Lin, T. Yang, L. Lang, C. Shan, H. Deng, W. Hu, F. Gao, Enhanced radiation tolerance of the Ni-Co-Cr-Fe high-entropy alloy as revealed from primary damage. Acta Mater. 196, 133-143 (2020). https://doi.org/10.1016/j.actamat.2020.06.027

86. J. Hu, H. Shen, M. Jiang, H. Gong, H. Xiao, Z. Liu, G. Sun, $\mathrm{X}$. Zu, A DFT study of hydrogen storage in high-entropy alloy
TiZrHfScMo. Nanomaterials 9(3), 461 (2019). https://doi.org/10. 3390/nano9030461

87 M. Widom, W.P. Huhn, S. Maiti, W. Steurer, Hybrid Monte Carlo/ molecular dynamics simulation of a refractory metal high entropy alloy. Metall. Mater. Trans. A 45(1), 196-200 (2014). https://doi. org/10.1007/s11661-013-2000-8

88. X. Liu, J. Zhang, J. Yin, S. Bi, M. Eisenbach, Y. Wang, Monte Carlo simulation of order-disorder transition in refractory high entropy alloys: a data-driven approach. Comput. Mater. Sci. 187, 110135 (2021). https://doi.org/10.1016/j.commatsci.2020.110135

89. M.M. Nygård, W.A. Sławiński, G. Ek, M.H. Sørby, M. Sahlberg, D.A. Keen, B.C. Hauback, Local order in high-entropy alloys and associated deuterides - a total scattering and Reverse Monte Carlo study. Acta Mater. 199, 504-513 (2020). https://doi.org/10.1016/j. actamat.2020.08.045

90. S.-P. Ju, I.J. Lee, H.-Y. Chen, Melting mechanism of Pt-Pd-RhCo high entropy alloy nanoparticle: an insight from molecular dynamics simulation. J. Alloy Compd. 858, 157681 (2021). https://doi.org/10.1016/j.jallcom.2020.157681

91. X. Zhou, W.A. Curtin, First principles study of the effect of hydrogen in austenitic stainless steels and high entropy alloys. Acta Mater. 200, 932-942 (2020). https://doi.org/10.1016/j.actamat. 2020.09.070

92. L. Zhang, Y. Shibuta, Inverse Hall-Petch relationship of highentropy alloy by atomistic simulation. Mater. Lett. 274, 128024 (2020). https://doi.org/10.1016/j.matlet.2020.128024

93. J. Xiao, C. Deng, Martensite transformation induced superplasticity and strengthening in single crystalline $\mathrm{CoNiCrFeMn} \mathrm{high}$ entropy alloy nanowires: a molecular dynamics study. Mater. Sci. Eng. A 793, 139853 (2020). https://doi.org/10.1016/j.msea.2020. 139853 